\title{
算子方程近似解的平均优化及其应用*
}

\section{王兴华 马 万}

(浙江大学数学系, 杭州 310028)

\begin{abstract}
摘要给出了在平均情形下, 算子方程近似解优化的一种度量, 得到一类算子方程 在此度量下优化的一般结果, 由此得到一类积分方程近似解优化的精确阶.
\end{abstract}

\section{关键词 Gauss 测度 积分 $n$ - 宽度 优化 算子方程}

设 $X$ 是一赋范线性空间, $Y$ 是 $X$ 的一个可分的子空间. $\mathcal{L}(X, Y)$ 表示 $X$ 到 $Y$ 的连续线 性算子 $H$ 的全体. $\mathcal{H}$ 是 $\mathcal{L}(X, Y)$ 的一个子集, 使得方程

$$
z=H z+f
$$

对任意的 $H \in \mathcal{H}$ 和 $f \in Y$ 有惟一解. 此类方程记为 $\Psi_{\mathcal{H}}^{Y}$.

近似解问题的优化是构造最优算法的基础. 一般来说，一个问题的优化总是与所使用的 度量有关. 一致度量是常用的, 但其他的度量, 如平均度量、概率度量等也是很有趣的 (见文 献 [1]). 就算子方程近似解的优化问题而言, 到目前为止, 已有许多一致度量下的工作 (见文献 $[2]$ ). 然而在平均情形下，此类问题的结果似不多见. 主要问题在于优化问题本身的艰深性以及 无适当的度量, 因此, 本文给出了在平均情形下刻画算子方程近似解优化的一种度量, 得到在 此度量下, 算子方程近似解优化的一般结果, 并由此解决了一类积分方程近似解的优化问题, 得到此类问题优化的精确阶.

\section{1 预备知识}

本文中所用的术语取自文献 [1]. 对 $\Psi_{\mathcal{H}}^{Y}$ 中方程, 定义解算子 $S: X \rightarrow X$ 如下:

$$
u=S f \text { 当且仅当 }(I-H) u=f \text {. }
$$

由文献 [3] 知, $S$ 是有界线性算子. 用 $\mathcal{B}(Y)$ 表示包含 $Y$ 中所有开集的 Borel 域, $\mu$ 是其上的 一个概率测度, $N: X \rightarrow \mathcal{R}^{n}$ 是信息算子, $\varphi$ 是使用信息 $N$ 的一个算法, 则 $\varphi$ 的平均误差定 义为下面的量:

$$
e^{\operatorname{avg}}(\varphi, N, S)=\int_{Y}\|S(f)-\varphi(N(f))\| \mu(d f) .
$$

用 $\operatorname{card}(N)$ 表示信息 $N$ 的基数, 称量

$$
\Theta_{n}^{\operatorname{avg}}\left(\Psi_{\mathcal{H}}^{Y}, X\right)=\inf _{N: \operatorname{card}(N) \leqslant n} \inf _{\varphi, \operatorname{using} N} e^{\operatorname{avg}}(\varphi, N, S)
$$


为在信息不超过 $n$ 的前提下，求解 $\Psi_{\mathcal{H}}^{Y}$ 中方程的最小误差. 在有的文献中，与 (3) 式类似的量 称为第 $n$ 个最小信息半径. 所谓算子方程在平均意义下的优化就是对量 (3) 的阶进行估计.

\section{2 主要定理及证明}

定理 1 设 $n=1,2, \cdots$, 则

$$
\Theta_{n}^{\operatorname{avg}}\left(\Psi_{\mathcal{H}}^{Y}, X\right) \asymp d_{n}^{(a)}(Y, X, \mu),
$$

其中 $a_{n} \asymp b_{n}$ 意味着 $a_{n} \ll b_{n}$ 且 $b_{n} \ll a_{n}$, 而 $a_{n} \ll b_{n}$ 表示存在数 $c$ 和 $n_{0} \in \mathbb{N}$, 使得对所有 $n \geqslant n_{0}$ 有 $a_{n} \leqslant c b_{n} ; d_{n}^{(a)}(Y, X, \mu)$ 表示 $Y$ 在 $X$ 中具测度 $\mu$ 的积分 $n$ - 宽度 (见文献 [4]).

证 设 $N$ 是基数不超过 $n$ 的任意算子, $\varphi_{N}^{s}$ 是基于信息 $N$ 的 $\mu$-样条, 则由文献 [1] 知, $S \varphi_{N}^{s}$ 是一个最优误差算法, 即

$$
\begin{aligned}
\inf _{\varphi, \text { using } N} e^{\operatorname{avg}}(\varphi, N, S) & =e^{\operatorname{avg}}\left(S \varphi_{N}^{s}, N, S\right) \\
& =\int_{Y}\left\|S(f)-S \varphi_{N}^{s}(N(f))\right\| \mu(d f) .
\end{aligned}
$$

另一方面, 置 $L=I-H$, 可以证明, 存在仅依赖于 $\|S\|$ 和 $\|L\|$ 的常数 $c$, 使得

$$
c^{-1}\|S(f)\| \leqslant\|f\| \leqslant c\|S(f)\|, \quad f \in X .
$$

事实上, 从 $S$ 的定义

$$
u=S f \Longleftrightarrow(I-H) u=f
$$

可以得到

$$
\|f\|=\|L u \mid \leqslant\| L\left\|_{X \rightarrow X}\right\| u\|=\| L\left\|_{X \rightarrow X}\right\| S(f) \| .
$$

注意到

$$
\|S(f)\| \leqslant\|S\|_{X \rightarrow X}\|f\|,
$$

故在 (6) 式中取 $c=\max \left\{\|S\|_{X \rightarrow X},\|L\|_{X \rightarrow X}\right\}$ 即得 (5) 式. 于是, 由 (4) 和 (5) 式可得

$$
\begin{aligned}
c^{-1} \int_{Y}\left\|f-\varphi_{N}^{s}(N(f))\right\| \mu(d f) & \leqslant \inf _{\varphi, \operatorname{using~} N} e^{\operatorname{avg}}(\varphi, N, S) \\
& \leqslant c \int_{Y}\left\|f-\varphi_{N}^{s}(N(f))\right\| \mu(d f) .
\end{aligned}
$$

设 $\Lambda_{n}(X)=\left\{A_{n}: X \rightarrow F_{n} \mid F_{n} \subset X, \operatorname{dim} F_{n}=n\right\}$, 则 (见文献 [4])

$$
d_{n}^{(a)}(Y, X, \mu)=\inf _{A_{n} \in \Lambda_{n}(X)} \int_{Y}\left\|f-A_{n}(f)\right\| \mu(d f) .
$$

于是, 从 (7) 和 (8) 式及 $\varphi_{N}^{s}$ 的最优性可得

$$
\begin{aligned}
c^{-1} \inf _{A_{n} \in \Lambda_{n}(X)} \int_{Y}\left\|f-A_{n}(f)\right\| \mu(d f) & \leqslant \inf _{\varphi, \text { using } N} e^{\operatorname{avg}}(\varphi, N, S) \\
& \leqslant c \inf _{A_{n} \in \Lambda_{n}(X)} \int_{Y}\left\|f-A_{n}(f)\right\| \mu(d f) .
\end{aligned}
$$

在 (9) 式的两边对基数不超过 $n$ 的信息算子 $N$ 取下确界即得定理 1 . 


\section{3 定理 1 的应用}

设 $L_{p}(1 \leqslant p<\infty)$ 是 $2 \pi$ 周期的 $p$ 次可积函数空间，其上的范数定义如下:

$$
\|f\|_{p}=\left((2 \pi)^{-1} \int_{-\pi}^{\pi}|f(x)|^{p} d x\right)^{\frac{1}{p}} .
$$

$L_{\infty}$ 表示 $2 \pi$ 周期的连续函数，其上的范数定义如下:

$$
\|f\|_{\infty}=\sup _{x}|f(x)| \text {. }
$$

设 $r>(1 / 2-1 / p)_{+}$, 其中 $(t)_{+}=t(t \geqslant 0) ;(t)_{+}=0(t<0)$. 考虑 Sobolev 类 $W_{2}^{r}([0,2 \pi])$, 其中的函数可以表示为下述的形式:

$$
f(x)=\sum_{k=-\infty}^{\infty} \alpha_{k} e_{k}(x), \quad e_{k}(x)=\exp (\mathrm{i} k t),
$$

且

$$
\alpha_{0}=0, \quad\|f\|_{W_{2}^{r}}=\left(\sum_{k=-\infty}^{\infty}|k|^{2 r}\left|\alpha_{k}\right|^{2}\right)^{1 / 2}<\infty .
$$

在 $W_{2}^{r}([0,2 \pi])$ 上赋以 Gauss 测度 $\mu=\mu_{a, s}$, 其中 $\mu_{a, s}(a>0, s>1)$ 均值为零, 具有相关 算子 $C_{\mu}$, 特征函数 $\left\{e_{k}\right\}_{-\infty}^{\infty}$ 和特征值 $\lambda_{k}=a|k|^{-s}$, 且满足关系: $C_{\mu} e_{k}=\lambda_{k} e_{k}$.

函数

$$
e_{m n}(s, t)=e_{m}(s) e_{n}(s) \quad(m, n \in \mathbb{Z}, \quad s, t \in[0,2 \pi))
$$

组成 $L_{2}\left([0,2 \pi]^{2}\right)$ 的一组正交基. 对 $r \geqslant 0$, Sobolev 空间 $W_{2}^{r}\left([0,2 \pi]^{2}\right)$ 定义为

$$
W_{2}^{r}\left([0,2 \pi]^{2}\right)=\left\{g \in L_{2}\left([0,2 \pi]^{2}\right):\|g\|_{W_{2}^{r}\left([0,2 \pi]^{2}\right)}=\sum_{m, n \in \mathbb{Z}}\left(1+m^{2}+n^{2}\right)^{r}\left(g, e_{m, n}\right)^{2}<\infty\right\},
$$

其中, $(\cdot, \cdot)$ 是 $L_{2}\left([0,2 \pi]^{2}\right)$ 中通常的内积.

令

$$
H=\int_{0}^{2 \pi} h(s, t) x(t) d t .
$$

用 $\Psi_{p}^{r}$ 表示形如

$$
x(s)-\int_{0}^{2 \pi} h(s, t) x(t) d t=y(s)
$$

的 Fredholm 积分方程类, 其中 $h \in W_{2}^{r}\left([0,2 \pi]^{2}\right), y \in W_{2}^{r}$, 且 $\left\|(I-H)^{-1}\right\|_{L_{p} \rightarrow L_{p}} \leqslant c$.

用 $L_{p}(1 \leqslant p \leqslant \infty), W_{2}^{r}$ 和 $\mu_{a, s}$ 分别代替定理 1 中的 $X, Y$ 和 $H$ 及 $\mu$, 并利用文献 [4] 中的 定理 2 可得

定理 2 设 $r>(1 / 2-1 / p)_{+}, s>1, \alpha>8 \ln 5 /(s-1) \cdot 2^{s}, n=2,3, \cdots$, 则

$$
\Theta_{n}^{\mathrm{avg}}\left(\Psi_{p}^{r}, L_{p}\right) \asymp n^{-\rho} .
$$

\section{4 注释: 一致和平均度量的比较}

现在考虑如下的问题是有趣的, 即就本文中的问题而言, 平均情形和最坏情形 (见文献 [5]) 哪种能给我们提供更多的信息呢? 下面就 Fredholm 积分方程近似解的优化问题对这两种情形 作一比较. 
其一, 在最坏情形下, 最小误差的阶的指数为 $r$ (见文献 [5]), 然而, 平均情形下为 $\rho=$ $r+\frac{1}{2}(s-1)(s>1)$, 后者依赖于 $s$. 由此可见, 当 $s$ 很大时, 随着 $n$ 趋于无穷, 平均情形下误 差趋于零的速度比最坏情形下要快得多. 特别对较小的 $r$, 这种差别是主要的.

其二, 在最坏情形下， $r$ 必须为正以保证方法的收玫，然而在平均情形下， $r$ 可以为 0 , 此 时仍可保证方法的收玫性.

这些好的信息都是基于 Gauss 测度的引进，使得函数类的光滑性有所增加. 由此可见，平 均情形下确实提供了较最坏情形下为多的信息.

\section{参考文献}

1 Traub J F, Woźniakowski H, Wasilkoeski G W. Information-based Complexity. Boston: Academic Press, INC, 1988

2 Pereverzev S V. Optimization of Methods for Approximation Solution of Operator Equations. Kiev: Institute of Mathematics of Ukr NAN, 1996

3 Kress R. Linear Integral Equations. Berlin: Springer-Verlag, 1989

4 Maiorov V E. Widths of Banach spaces with Gaussian measures. Dokl Akad Hauk USSR, 1992, 323(2): $233 \sim 237$

5 Werschulz A G. What is the complexity of the Fredholm problem of the second kind. J Integral Equations Appl, 1984, 9: 213 241 